\title{
Algorithms for the Detection of a Signal with Unknown Amplitude and Duration against White Noise
}

\author{
Yury E. Korchagin \\ Voronezh State University \\ 1, University sq., Voronezh, 394006, Russia \\ Oleg V. Chernoyarov* \\ National Research Institute "MPEI" \\ 14, Krasnokazarmennaya st., Moscow, 111250, Russia \\ *Corresponding author
}

\begin{abstract}
We synthesized the quasi-likelihood and maximum likelihood algorithms for the detection of a free-form signal with unknown amplitude and duration against Gaussian white noise. We illustrated the methods for calculation of the characteristics of their operating effectiveness and also we found the analytical expressions for false-alarm and missing probabilities of the considered detectors.
\end{abstract}

Keywords-free-form signal; detection; maximum likelihood method; parametrical prior uncertainty; false-alarm and missing probabilities

\section{INTRODUCTION}

The problem of the detection of information signals with unknown power and time parameters is vital in radio physics and radio engineering. So, in works $[1,2]$ the reception of a rectangular pulse with unknown duration against white noise was considered. In [3] algorithms of reception of a free-form pulse with unknown duration were studied. However, in a number of practical applications the received signal amplitude is also unknown. In work [4] algorithms of reception of a rectangular pulse with unknown duration and amplitude were synthesized. The asymptotic expressions for detection error probabilities, and for distribution functions and variances of joint maximum likelihood estimates of a signal amplitude and duration were found. In the present work we carry out the synthesis and analysis of the algorithms for the detection of a free-form pulse with unknown duration and amplitude.

\section{PROBLEM STATEMENT}

We suppose that the signal

$$
s\left(t, a_{0}, \tau_{0}\right)= \begin{cases}a_{0} f(t), & 0 \leq t \leq \tau_{0}, \\ 0, & t<0, t>\tau_{0}\end{cases}
$$

is to be detected against Gaussian white noise $n(t)$ with onesided spectral density $N_{0}$. Thus, the realization $\xi(t)$ is observed over the time interval $[0, T]$, and it can be by noise

\author{
Alexandra V. Salnikova \\ Voronezh State University of Architecture and Civil \\ Engineering \\ 84, 20th anniversary of October st., Voronezh, Russia
}

\author{
Boris I. Shakhtarin \\ Bauman Moscow State University \\ 5, Baumanskaya 2-ya st., Moscow, Russia
}

only: $\xi(t)=n(t)$ or by additive mix of a signal and noise: $\xi(t)=s\left(t, a_{0}, \tau_{0}\right)+n(t)$. In Eq. (1) $a_{0}, \tau_{0}$ are the designations of the signal amplitude and duration accordingly, and $f(t)$ is the function describing the form of a signal.

Let us assume that the signal duration possesses the value within a prior interval $\tau \in\left[T_{1}, T_{2}\right]$, and function $f(t)$ is satisfied to a condition $\max f(t)=1, t \in\left[0, T_{2}\right]$. With observable realization $\xi(t)$ and prior information, it is necessary to made the decision on presence or absence of a useful signal (1).

If the useful signal (1) amplitude and duration are a priori known, then it is possible to apply optimal detection algorithm $[2,5,6]$ according to which a receiver forms the logarithm of the functional of likelihood ratio (FLR)

$$
L_{0}=\frac{2 a_{0}}{N_{0}} \int_{0}^{\tau_{0}} \xi(t) f(t) d t-\frac{a_{0}^{2}}{N_{0}} \int_{0}^{\tau_{0}} f^{2}(t) d t
$$

The decision on signal presence or absence is made as a result of the comparison of the generated value (2) with a threshold chosen according to the accepted optimality criterion. However, under unknown signal parameters $a_{0}$ and $\tau_{0}$ we have a prior uncertainty concerning amplitude and duration. In this case the logarithm of FLR is a function of two variables [2, 6]

$$
L(a, \tau)=\frac{2 a}{N_{0}} \int_{0}^{\tau} \xi(t) f(t) d t-\frac{a^{2}}{N_{0}} \int_{0}^{\tau} f^{2}(t) d t
$$

depending on current values $a$, $\tau$ of unknown parameters $a_{0}$, $\tau_{0}$. Correspondingly, a number of detection algorithms (probably, nonoptimal) can be synthesized by substituting some specific levels of $a$ and $\tau$ in Eq. (3). These levels can be fixed 
or they can be determined by the realization of the observable data, as it is considered below.

\section{QUASI-LIKELIHOOD DETECTION ALGORITHM}

The simplest way for the overcoming of the prior uncertainty concerning amplitude and duration is the application of the quasi-likelihood (QL) detection algorithm. QL receiver forms the logarithm of FLR for some expected (predictable) amplitude $a^{*}$ and duration $\tau^{*} \in\left[\mathrm{T}_{1}, \mathrm{~T}_{2}\right]$, i.e.

$$
L_{1}=L\left(a^{*}, \tau^{*}\right)=\frac{2 a^{*}}{N_{0}} \int_{0}^{\tau^{*}} \xi(t) f(t) d t-\frac{a^{* 2}}{N_{0}} \int_{0}^{\tau^{*}} f^{2}(t) d t
$$

The value (4) is compared to a threshold $h$ and a decision is made on presence or absence of a signal in observable realization.

In order to determine the QL algorithm performance, we find the analytical expressions for false-alarm and missing probabilities $[2,5]$. It is obvious that amplitude and duration ignorance influences detection efficiency. Therefore, we put into consideration the values characterizing the amplitude detuning $\Delta_{a}=a^{*} / a_{0}, \delta_{a}=\left(a^{*}-a_{0}\right) / a_{0}=\Delta_{a}-1$ and duration detuning $\Delta_{\tau}=\tau^{*} / \tau_{0}, \delta_{\tau}=\left(\tau^{*}-\tau_{0}\right) / \tau_{0}=\Delta_{\tau}-1$, so

$$
a^{*}=a_{0}\left(1+\delta_{a}\right)=a_{0} \Delta_{a}, \tau^{*}=\tau_{0}\left(1+\delta_{\tau}\right)=\tau_{0} \Delta_{\tau} .
$$

Substituting Eqs. (5) in Eq. (4) we have

$$
L_{1}=\frac{2 a_{0}}{N_{0}} \Delta_{a} \int_{0}^{\tau_{0} \Delta_{\tau}} \xi(t) f(t) d t-\frac{a_{0}^{2} \Delta_{a}^{2}}{N_{0}} \int_{0}^{\tau_{0} \Delta_{\tau}} f^{2}(t) d t
$$

Let us designate $L_{1}=L_{11}$ as the logarithm of FLR, if a useful signal is present in the received realization, and $L_{1}=L_{10}$ - if a useful signal is absent. The random variables $L_{11}, L_{10}$ are Gaussian being linear transformations from Gaussian white noise. Therefore, it is enough to find their mathematical expectations $S_{11}=\left\langle L_{11}\right\rangle, \quad S_{10}=\left\langle L_{10}\right\rangle$ and dispersions $D_{11}=\left\langle\left(L_{11}-S_{11}\right)^{2}\right\rangle, \quad D_{10}=\left\langle\left(L_{10}-S_{10}\right)^{2}\right\rangle$ for full statistical description. Carrying out averaging over all the realizations of the observable data we obtain

$$
\begin{gathered}
S_{11}=z_{0}^{2} \Delta_{a}\left[1+d\left(\min \left(1, \Delta_{\tau}\right)\right)\right]-z_{0}^{2} \Delta_{a}^{2}\left[1+d\left(\Delta_{\tau}\right)\right] / 2, \\
S_{10}=-z_{0}^{2} \Delta_{a}^{2}\left[1+d\left(\Delta_{\tau}\right)\right] / 2, D_{11}=D_{10}=D=z_{0}^{2} \Delta_{a}^{2}\left[1+d\left(\Delta_{\tau}\right)\right],
\end{gathered}
$$

where $z_{0}^{2}=\left(2 a_{0}^{2} / N_{0}\right) \int_{0}^{\tau_{0}} f^{2}(t) d t$ is the output signal-to-noise ratio $(\mathrm{SNR})[2,6]$ for optimal receiver (2), and $d(x)=\int_{\tau_{0}}^{\tau_{0} x} f^{2}(t) d t / \int_{0}^{\tau_{0}} f^{2}(t) d t$ is the generalized detuning on duration.

Using statistical characteristics (6) of the random variables $L_{11}, L_{10}$ we write down exact expressions for false-alarm probability:

$$
\begin{gathered}
\alpha_{1}=P\left[L_{10}>h\right]=1-P\left[L_{10}<h\right]= \\
=1-\Phi\left[h / z_{0} \Delta_{a} \sqrt{1+d\left(\Delta_{\tau}\right)}+\left(z_{0} \Delta_{a} / 2\right) \sqrt{1+d\left(\Delta_{\tau}\right)}\right]
\end{gathered}
$$

and missing probability:

$$
\begin{gathered}
\beta_{1}=P\left[L_{11}<h\right]=F_{11}(h)=\Phi \mid h / z_{0} \Delta_{a} \sqrt{1+d\left(\Delta_{\tau}\right)}- \\
\left.-z_{0}\left(1+d\left(\min \left(1, \Delta_{\tau}\right)\right)\right) / \sqrt{1+d\left(\Delta_{\tau}\right)}+\left(z_{0} \Delta_{a} / 2\right) \sqrt{1+d\left(\Delta_{\tau}\right)}\right] .
\end{gathered}
$$

Here $\Phi(x)=\int_{-\infty}^{x} \exp \left(-t^{2} / 2\right) d t / \sqrt{2 \pi}$ is the probability integral.

If expected amplitude and duration coincide with their true values, i.e. $\tau_{0}=\tau^{*}$ and $a_{0}=a^{*}$, then QL detector coincides with optimal detector for a priori known signal, which possesses characteristics [5]

$$
\alpha_{0}=1-\Phi\left(h / z_{0}+z_{0} / 2\right), \beta_{0}=\Phi\left(h / z_{0}-z_{0} / 2\right)
$$

Eqs. (7), (8) and (9) allow us to define the losses in efficiency of QL detector (4) in comparison with optimal detector (2) owing to a departure of $a^{*}$ from $a_{0}$ and $\tau^{*}$ from $\tau_{0}$.

\section{Amplitude AdAPTATION AlgORITHM}

In order to increase detection efficiency, QL amplitude adaptation algorithm can be used according to which the logarithm of FLR is formed for expected duration $\tau^{*}$ and its maximization on amplitude is carried out:

$$
L_{2}=\max _{a} L\left(a, \tau^{*}\right)=\max _{a} L_{2}(a)
$$

The decision on signal presence or absence is made by comparing the value (10) with a threshold $h$. Maximization of the logarithm of FLR (3) on amplitude can be carried out analytically. Really, it is easily seen that the function $L_{2}(a)=L\left(a, \tau^{*}\right)$ tops, if

$$
a=\int_{0}^{\tau^{*}} \xi(t) f(t) d t / \int_{0}^{\tau^{*}} f^{2}(t) d t
$$

Substituting Eq. (11) in Eq. (10) we obtain the following expression for the decision statistics: 


$$
L_{2}=\left(\int_{0}^{\tau^{*}} \xi(t) f(t) d t / \sqrt{N_{0} \int_{0}^{\tau^{*}} f^{2}(t) d t}\right)^{2}
$$

While analyzing the synthesized algorithm, we find the distribution function of a random variable (12). We designate $L_{2}=L_{21}$ in case of the signal (1) presence, and $L_{2}=L_{20}$, when the signal (1) is absent. In Eq. (12) the value $\kappa=\int_{0}^{\tau^{*}} \xi(t) f(t) d t / \sqrt{N_{0} \int_{0}^{\tau^{*}} f^{2}(t) d t}$ is squared, which is Gaussian random one with mathematical expectation $m=0$ (when the signal is absent) or $m=a_{0} \int_{0}^{\min \left(\tau_{0}, \tau^{*}\right)} \xi(t) f^{2}(t) d t / \sqrt{N_{0} \int_{0}^{\tau^{*}} f^{2}(t) d t} \quad$ (when the signal is present) and dispersion $\sigma^{2}=1 / 2$. Hence, for the distribution function of the random variable $L_{2}$ (12) we have

$$
F_{L_{2}}(y)=P\left[L_{2}<y\right]=P[-\sqrt{y}<\kappa<\sqrt{y}]=F_{\kappa}(\sqrt{y})-F_{\kappa}(-\sqrt{y})
$$

where $F_{\kappa}(y)$ is distribution function of the Gaussian random variable $\kappa$. Using Eq. (13) we now write down exact expressions for false-alarm probability:

$$
\alpha_{2}=1-P\left[L_{20}<h\right]=2[1-\Phi(2 \sqrt{h})]
$$

and missing probability:

$$
\begin{gathered}
\beta_{2}=P\left[L_{21}<h\right]=\Phi\left[2 \sqrt{h}-\frac{z_{0}\left(1+d\left(\min \left(1, \Delta_{\tau}\right)\right)\right)}{\sqrt{\left(1+d\left(\Delta_{\tau}\right)\right) / 2}}\right]+ \\
+\Phi\left[2 \sqrt{h}+\frac{z_{0}\left(1+d\left(\min \left(1, \Delta_{\tau}\right)\right)\right)}{\sqrt{\left(1+d\left(\Delta_{\tau}\right)\right) / 2}}\right]-1 .
\end{gathered}
$$

Comparing Eqs. (14), (15) and Eqs. (7), (8) we can determine a detection efficiency gain resulting from the amplitude adaptation realization.

\section{DURATION ADAPTATION ALGORITHM}

Other way of improvement of the detection quality in comparison with Eq. (4) is the use of QL detection algorithm with adaptation on duration. The receiver forms the logarithm of FLR (3) for expected amplitude, while maximization on duration is carried out

$$
L_{3}=\max _{\tau} L\left(a^{*}, \tau\right)=\max _{\tau} L_{3}(\tau)
$$

The decision on signal presence or absence is made as a result of comparison of value (16) with a threshold $h$. We designate

$$
L_{3}(\tau)=L\left(a^{*}, \tau\right)=\frac{2 a^{*}}{N_{0}} \int_{0}^{\tau} \xi(t) f(t) d t-\frac{a^{* 2}}{N_{0}} \int_{0}^{\tau} f^{2}(t) d t
$$

Let $L_{3}(\tau)=L_{30}(\tau)$, if the signal (1) is absent, and $L_{3}(\tau)=L_{31}(\tau)$, if the signal (1) is present in the received realization. According to Eq. (17), the random processes $L_{30}(\tau), L_{31}(\tau)$ are Gaussian. Therefore, it is enough to find their mathematical expectations and covariance functions for the full statistical description. Carrying out averaging over all the realizations of the observable data we get

$$
\begin{gathered}
S_{30}(\tau)=\left\langle L_{30}(\tau)\right\rangle=-\Delta_{a}^{2} q(\tau) / 2, \\
S_{31}(\tau)=\left\langle L_{31}(\tau)\right\rangle=\Delta_{a} q\left(\min \left(\tau, \tau_{0}\right)\right)-\Delta_{a}^{2} q(\tau) / 2, \\
K\left(\tau_{1}, \tau_{2}\right)=\left\langle\left[L_{3 i}\left(\tau_{1}\right)-\left\langle L_{3 i}\left(\tau_{1}\right)\right\rangle\right]\left[L_{3 i}\left(\tau_{2}\right)-\left\langle L_{3 i}\left(\tau_{2}\right)\right\rangle\right]\right\rangle= \\
=\Delta_{a}^{2} q\left(\min \left(\tau_{1}, \tau_{2}\right)\right), \quad i=0,1 .
\end{gathered}
$$

Here $q(\tau)=\left(2 a_{0}^{2} / N_{0}\right) \int_{0}^{\tau} f^{2}(t) d t$ is output SNR for a signal with duration $\tau$.

In Eq. (17) we move to a new variable $\lambda=q(\tau)$. As function $q(\tau)$ is nonnegative and monotonic, the following equality $q\left(\min \left(\tau_{1}, \tau_{2}\right)\right)=\min \left(q\left(\tau_{1}\right), q\left(\tau_{2}\right)\right)$ is valid. Then, we can present the decision statistics (17) as function of the variable $\lambda$ in the form of

$$
L_{31}(\tau)=L_{31}[\tau(\lambda)]=\mu_{31}(\lambda)=\Delta_{a} \min \left(\lambda, \lambda_{0}\right)-\Delta_{a}^{2} \lambda / 2+v(\lambda)
$$

with the signal (1) present and

$$
L_{30}(\tau)=L_{30}[\tau(\lambda)]=\mu_{30}(\lambda)=-\Delta_{a}^{2} \lambda / 2+v(\lambda)
$$

with the signal (1) absent. Here $\lambda_{0}=q\left(\tau_{0}\right)$, and $v(\lambda)$ is Gaussian random process with zero mathematical expectation and covariance function

$$
B\left(\lambda_{1}, \lambda_{2}\right)=\Delta_{a}^{2} \min \left(\lambda_{1}, \lambda_{2}\right),
$$

and $\tau(\lambda)$ is determined from solution of equation $q(\tau)=\lambda$.

By definition the false-alarm probability $\alpha_{3}$ can be expressed in terms of the probability $P_{0}(h)$ of threshold $h$ uncrossing by the random process $\mu_{30}(\lambda)$, that is $\alpha_{3}=1-P\left\{-\infty<\mu_{30}(\lambda)<h, \lambda \in\left[\Lambda_{1}, \Lambda_{2}\right]\right\}=1-P_{0}(h)$

Similarly, the missing probability is equal to probability $P_{1}(h)$ of the threshold $h$ uncrossing by the random process $\mu_{31}(\lambda): \beta_{3}=P\left\{-\infty<\mu_{31}(\lambda)<h, \lambda \in\left[\Lambda_{1}, \Lambda_{2}\right]\right\}=P_{1}(h)$. Here it is designated as 


$$
P_{i}(h)=P\left\{-\infty<\mu_{3 i}(\lambda)<h, \lambda \in\left[\Lambda_{1}, \Lambda_{2}\right]\right\}
$$

In order to find the functions (19), we use the procedure stated in $[7,8]$. According to Eq. (18), the random process $\mu_{3 i}(\lambda)$ is Markov one with drift $k_{1 i}$ and diffusion $k_{2 i}$ coefficients

$$
k_{1 i}= \begin{cases}i \Delta_{a}-\Delta_{a}^{2} / 2, & \lambda \leq \lambda_{0}, \\ -\Delta_{a}^{2} / 2, & \lambda>\lambda_{0}, \quad k_{2 i}=\Delta_{a}^{2}, \quad i=0,1\end{cases}
$$

Therefore, we can write down $[7,8]$

$$
P_{i}(h)=P\left\{\mu_{3 i}(\lambda)>0, \lambda \in\left[\Lambda_{1}, \Lambda_{2}\right]\right\}=\int_{0}^{\infty} W_{i}\left(y, \Lambda_{2}\right) d y
$$

Here $W_{i}(y, \lambda)$ is a solution of the direct Fokker-PlanckKolmogorov equation [7-9]

$$
\frac{\partial W_{i}(y, \lambda)}{\partial \lambda}+\frac{\partial}{\partial y}\left[k_{1 i} W_{i}(y, \lambda)\right]-\frac{1}{2} \frac{\partial^{2}}{\partial y^{2}}\left[k_{2 i} W_{i}(y, \lambda)\right]=0
$$

with boundary conditions $W_{i}(y=0, \lambda)=W_{i}(y=\infty, \lambda)=0$ and starting condition

$$
\left.W_{i}(y, \lambda)\right|_{\lambda=\Lambda_{1}}=\frac{1}{\Delta_{a} \sqrt{2 \pi \Lambda_{1}}} \exp \left[-\frac{\left(y-h+i \Delta_{a} \Lambda_{1}-\Delta_{a}^{2} \Lambda_{1} / 2\right)^{2}}{2 \Delta_{a}^{2} \Lambda_{1}}\right]
$$

Applying a reflection method with sign inversion, we find the solution of Eq. (22) with coefficients (20) separately for the cases $\lambda \in\left[\Lambda_{1}, \lambda_{0}\right]$ and $\lambda \in\left[\lambda_{0}, \Lambda_{2}\right]$, as it is described in [7]. Further, following [8] and substituting the found solutions in Eq. (21) we obtain expressions for false-alarm and missing probabilities in the form of

$$
\begin{aligned}
\alpha_{3}=1 & -\frac{1}{\Delta_{a} \sqrt{2 \pi \Lambda_{1}}} \int_{0}^{\infty} \exp \left[-\left(h-\xi+\Delta_{a}^{2} \Lambda_{1} / 2\right)^{2} / 2 \Delta_{a}^{2} \Lambda_{1}\right] \times \\
& \times\left\{\Phi\left(\Delta_{a} \sqrt{\Lambda_{2}-\Lambda_{1}} / 2+\xi / \Delta_{a} \sqrt{\Lambda_{2}-\Lambda_{1}}\right)-\right. \\
\left.-\exp (-\xi) \Phi\left(\Delta_{a} \sqrt{\Lambda_{2}-\Lambda_{1}} / 2-\xi / \Delta_{a} \sqrt{\Lambda_{2}-\Lambda_{1}}\right)\right\} d \xi &
\end{aligned}
$$

$$
\begin{gathered}
\beta_{3}=\frac{\exp \left(\left(\Lambda_{1}-\lambda_{0}\right)\left(1-\Delta_{a} / 2\right)^{2} / 2\right]}{2 \pi \Delta_{a}^{2} \sqrt{\Lambda_{1}\left(\lambda_{0}-\Lambda_{1}\right)}} \int_{0}^{\infty} \int_{0}^{\infty} d \xi d \xi_{1} \exp \left[\left(\xi-\xi_{1}\right) \times\right. \\
\left.\times\left(1 / \Delta_{a}-1 / 2\right)-\left(h-\xi-\Delta_{a} \Lambda_{1}+\Delta_{a}^{2} \Lambda_{1} / 2\right)^{2} / 2 \Delta_{a}^{2} \Lambda_{1}\right] \times \\
\times\left[\Phi\left(\Delta_{a} \sqrt{\Lambda_{2}-\lambda_{0}} / 2+\xi_{1} / \Delta_{a} \sqrt{\left(\Lambda_{2}-\lambda_{0}\right)}\right)-\right. \\
\left.-\exp \left(-\xi_{1}\right) \Phi\left(\Delta_{a} \sqrt{\Lambda_{2}-\lambda_{0}} / 2-\xi_{1} / \Delta_{a} \sqrt{\left(\Lambda_{2}-\lambda_{0}\right)}\right)\right] \times \\
\times\left\{\exp \left[-\left(\xi_{1}-\xi\right)^{2} / 2 \Delta_{a}^{2}\left(\lambda_{0}-\Lambda_{1}\right)\right]-\exp \left[-\left(\xi_{1}+\xi\right)^{2} / 2 \Delta_{a}^{2}\left(\lambda_{0}-\Lambda_{1}\right)\right]\right\} .
\end{gathered}
$$

Comparing Eqs. (23), (24) and (7), (8) we can determine detection efficiency gain due to the application of the adaptation on duration and then make the decision on the expediency of such adaptation application.

\section{MAXIMUM LIKELIHOOD DETECTION ALGORITHM}

In order to improve a detection quality, it is possible to apply a maximum likelihood detection algorithm according to which the receiver carries out a maximization of the logarithm of FLR (3) on both duration and amplitude:

$$
L_{4}=\max _{\tau} L_{4}(\tau), \quad L_{4}(\tau)=\max _{a} L(a, \tau)
$$

The decision on signal presence or absence is made comparing the value $L_{4}$ with a threshold $h$. Similarly to Eqs. (11), (12) the maximization of the logarithm of FLR (3) on variable $a$ can be carried out analytically. As a result we have

$$
L_{4}(\tau)=\frac{1}{N_{0}}\left(\int_{0}^{\tau} \xi(t) f(t) d t\right)^{2} / \int_{0}^{\tau} f^{2}(t) d t
$$

Eq. (25) defines the detector structure. The detector should form the random process (25) for all possible duration values and find its maximum. Comparing this maximum with a threshold the decision is made on presence or absence of the useful signal.

Let us find the detection performances for the algorithm working on the basis of the Eq. (25). For this purpose we consider the random process $M(\tau)=\int_{0}^{\tau} \xi(t) f(t) d t$, which is Gaussian one with the zero mathematical expectation in the signal absence and the mathematical expectation

$$
S_{M}(\tau)=\langle M(\tau)\rangle=a_{0} \int_{0}^{\min \left(\tau, \tau_{0}\right)} f^{2}(t) d t=a_{0} \rho\left(\min \left(\tau, \tau_{0}\right)\right)
$$

in the signal presence. Here it is designated

$$
\rho(\tau)=\int_{0}^{\tau} f^{2}(t) d t
$$


Covariance function of the process $M(\tau)$ is of the form

$$
\begin{aligned}
K_{M}\left(\tau_{1}, \tau_{2}\right)=\langle & {\left.\left[M\left(\tau_{1}\right)-\left\langle M\left(\tau_{1}\right)\right\rangle\right]\left[M\left(\tau_{2}\right)-\left\langle M\left(\tau_{2}\right)\right\rangle\right]\right\rangle=} \\
& =\left(N_{0} / 2\right) \rho\left(\min \left(\tau_{1}, \tau_{2}\right)\right) .
\end{aligned}
$$

We move to the new variable $r=\rho(\tau), \quad r \in\left[R_{1}, R_{2}\right]$, $R_{1}=\rho\left(T_{1}\right), R_{2}=\rho\left(T_{2}\right)$ in Eq. (25). As the function $\rho(\tau)$ is nonnegative and monotonic, we have $\rho\left(\min \left(\tau_{1}, \tau_{2}\right)\right)=\min \left(\rho\left(\tau_{1}\right), \rho\left(\tau_{2}\right)\right)$. Then, the decision statistics (25) as function of the variable $r$ can be presented in a kind of

$$
L_{4}(r)=\chi^{2}(r) / N_{0} r
$$

Here $\chi(r)$ is Gaussian random process with covariance function

$$
K_{\chi}\left(r_{1}, r_{2}\right)=\left(N_{0} / 2\right) \min \left(r_{1}, r_{2}\right)
$$

and also zero mathematical expectation in the signal absence and mathematical expectation

$$
S_{\chi}(r)=a_{0} \min \left(r, r_{0}\right), \quad r_{0}=\rho\left(\tau_{0}\right)
$$

in the signal presence. From Eqs. (28), (29) follows that the random process $\chi(r)$ can be presented as $\chi_{0}(r)=\omega(r)$ in the signal absence and $\chi_{1}(r)=a_{0} \min \left(r, r_{0}\right)+\omega(r)$ in the signal presence, where $\omega(r)$ is Wiener process [9].

In order to determine the false-alarm probability for $\mathrm{ML}$ detector, we move to the new variable $\theta=\ln r, \theta \in\left[\theta_{1}, \theta_{2}\right]$, $\theta_{1}=\ln R_{1}, \theta_{2}=\ln R_{2}$ in Eq. (27). According to [9], the function $L_{4}(\theta)=L_{4}(r(\theta))$ is Markov random process which satisfies to the stochastic differential Ito's equation $d L_{4}(\theta)=-L_{4}(\theta) d \theta+\sqrt{2 L_{4}(\theta)} d \omega(\theta)$. It is known [2] that with the increase of the threshold $h$ and the length of the prior interval $\left[\theta_{1}, \theta_{2}\right]$ the probability $P\left[\max L_{4}(\theta)<h\right]$ is limited to

$$
P\left[\max L_{4}(\theta)<h\right] \rightarrow \exp \left[-\sqrt{h / \pi}\left(\theta_{2}-\theta_{1}\right) \exp (-h)\right]
$$

Returning to the variable $r=\exp (\theta)$ in the last formula we have for large values of the threshold $h$ and relation $R_{2} / R_{1}$ :

$$
P\left[\max L_{4}(r)<h\right] \approx\left(R_{1} / R_{2}\right)^{\sqrt{h / \pi} \exp (-h)} .
$$

From here we obtain for false-alarm probability: $\alpha_{4}=P\left[\max L_{4}(r)>h\right] \approx 1-P\left[L_{4}(r)<h\right]$. Taking into account that the false-alarm probability should be never-decreasing function of a threshold, we specify last formula as

$$
\alpha_{4} \approx \begin{cases}1-\left(R_{1} / R_{2}\right)^{\sqrt{h / \pi} \exp (-h)}, & h \geq 1 / 2, \\ 1, & h<1 / 2 .\end{cases}
$$

Now, moving to the variable $\tau=\tau(r)$ in Eq. (30), where $\tau(r)$ is the solution of equation $r=\rho(\tau)$ (26), the following final asymptotic expression for the false-alarm probability can be written down

$$
\alpha_{4} \approx \begin{cases}1-\left(\int_{0}^{T_{1}} f^{2}(t) d t / \int_{0}^{T_{2}} f^{2}(t) d t\right)^{\sqrt{h / \pi} \exp (-h)}, & h \geq 1 / 2, \\ 1, & h<1 / 2\end{cases}
$$

with large values of a threshold and relation $T_{2} / T_{1}$.

Let us pass to the determining of the missing probability. In the signal presence at the receiver input the decision statistics (27) has the appearance

$$
L_{4}(r)=z_{0}^{2} \frac{\min ^{2}(1, l)}{2 l}+z_{0} \frac{\min (1, l)}{l} \omega(l)+\frac{\omega^{2}(l)}{2 l},
$$

where $l=r / r_{0}, r_{0}=\int_{0}^{\tau_{0}} f^{2}(t) d t$, and $z_{0}$ is defined the same way as in Eqs. (6).

With large SNR we can neglect the last summand in Eq. (32) and write down the approximate equality

$$
L_{4}(r) \approx z_{0}^{2} \min ^{2}(1, l) / 2 l+z_{0} \min (1, l) \omega(l) / l
$$

This function is Markov random process with drift $k_{1}$ and diffusion $k_{2}$ coefficients having the appearance of

$$
k_{1} \approx \frac{z_{0}^{2}}{2 r_{0}} \begin{cases}1, & \tau<\tau_{0}, \\ -1 / l^{2}, & \tau>\tau_{0}, \quad k_{2}=2\left|k_{1}\right|\end{cases}
$$

Markovian properties of the process (33) allow finding the auxiliary function

$$
F(u, v, R)=P\left[\begin{array}{cc}
L_{4}(r)<u, L_{4}(r)<v \\
R_{1}<r<R & R<r<R_{2}
\end{array}\right],
$$

through which the missing probability can be easily expressed [8]

$$
\beta_{4}=P\left[\max L_{4}(r)<h\right]=F\left(h, h, R_{2}\right)
$$


Following [8], for function (35) we can write down $F(u, v, R)=\int_{-\infty}^{u} W\left(x, R_{2}\right) d x$, where $W(x, R)$ is a solution of the Fokker-Planck-Kolmogorov equation (22) with coefficients (34), boundary conditions $\left.W(x, r)\right|_{x=u}=0, r \in\left[R_{1}, R\right]$ and $\left.W(x, r)\right|_{x=v}=0 \quad, \quad r \in\left[R, R_{2}\right], \quad$ and starting condition $\left.W(x, r)\right|_{r=R_{1}}=W_{1}(x)$. Here $W_{1}(x)$ is the probability density of the random value $L_{4}\left(R_{1}\right)$ (33). By solving this equation, as it is described in [8], we find the function (35), which we substitute in Eq. (36) and obtain the asymptotic expression for the missing probability in the form of

$$
\begin{gathered}
\beta_{4} \approx \int_{0}^{\infty} d \xi_{1} \int_{0}^{h} d \xi \frac{\operatorname{ch}\left(z_{0} \sqrt{2 \rho\left(T_{1}\right)(h-\xi) / \rho\left(\tau_{0}\right)}\right)}{\pi z_{0} \sqrt{2(h-\xi)\left[1-\rho\left(T_{1}\right) / \rho\left(\tau_{0}\right)\right.}}\{1- \\
\left.-\exp \left[-\frac{2 \xi \xi_{1}}{z_{0}^{2}\left(1-\rho\left(T_{1}\right) / \rho\left(\tau_{0}\right)\right)}\right]\right\}\left\{\Phi\left(\frac{z_{0}^{2}\left[1-\rho\left(\tau_{0}\right) / \rho\left(T_{2}\right)\right] / 2+\xi_{1}}{z_{0} \sqrt{1-\rho\left(\tau_{0}\right) / \rho\left(T_{2}\right)}}\right)-\right. \\
\times \exp \left(-\xi_{1}\right) \Phi\left(\frac{\left.z_{0}^{2}\left[1-\rho\left(\tau_{0}\right) / \rho\left(T_{2}\right)\right] / 2-\xi_{1}\right)}{\left.\left.z_{0} \sqrt{1-\rho\left(\tau_{0}\right) / \rho\left(T_{2}\right)}\right)\right\} \times}\right. \\
\times \exp \left\{\xi-h-\frac{z_{0}^{2} \rho\left(T_{1}\right)}{2 \rho\left(\tau_{0}\right)}-\frac{\left[\xi_{1}-\xi+z_{0}^{2}\left(1-\rho\left(T_{1}\right) / \rho\left(\tau_{0}\right)\right) / 2\right.}{2 z_{0}^{2}\left[1-\rho\left(T_{1}\right) / \rho\left(\tau_{0}\right)\right]}\right\} .
\end{gathered}
$$

Comparing Eqs. (31), (37) and Eqs. (7), (8), (14), (15), (23), (24) we can determine an efficiency gain of the maximum likelihood detector in comparison with QL ones and estimate the expediency of its application in each specific case.

\section{CONCLUSION}

The obtained results allow making an informed choice of the detector with accounting for the complexity of its hardware implementation and detection efficiency. The simplest algorithm in terms of the hardware implementation is QL algorithm (4). Really, it is only required to form a random variable for the decision making here, and this variable linearly depends on the realization of the observable data. However, QL algorithm (4) is characterized by the lowest detection efficiency. In order to increase the detection efficiency, it is possible to use either QL detector (12) with adaptation on amplitude or QL detector (17) with adaptation on duration. The first of them should generate the random variable which nonlinearly depends on the realization of the observable data, the second detector should search an absolute maximum of the random process which is linear transformation from the realization of the observable data. The maximum likelihood detection algorithm (25) is the most effective and complicated one in the implementation. Indeed, for the decision making it is required to find an absolute maximum of the random process nonlinearly depending on the realization of the observable data. But this complication can be considered as insignificant in many cases.

\section{ACKNOWLEDGMENT}

The reported study was supported by Russian Science Foundation (research project No. 14-49-00079).

\section{REFERENCES}

[1] A.P. Trifonov, "Receiving a signal with unknown duration against white noise", Radio Engineering and Electronic Physics, vol. 22, No. 1, 1977, pp. 90-98.

[2] A.P. Trifonov, Yu.S. Shinakov, Joint Discrimination of Signals and Estimation of Their Parameters against BackGround [in Russian]. Radio i Svyaz', Moscow, 1986.

[3] A.P. Trifonov, Yu.E. Korchagin, "Receiving a signal with unknown duration", Izvestiya Vysshikh Uchebnykh Zavedenij. Radiofizika, vol. 45, No 7, 2002, pp. 625-637.

[4] A.P. Trifonov, V.K. Buteiko, "Reception of a signal of unknown amplitude and duration on a background of white noise", Izvestiya Vysshikh Uchebnykh Zavedenij. Radioelektronika, vol. 27, No 8, 1981, pp. 28-34..

[5] V.I. Tikhonov, Optimal Signal Receiption [in Russian]. Radio i Svyaz', Moscow, 1983.

[6] Signal Detection Theory [in Russian], Edited by P.A. Bakut, Radio i Svyaz', Moscow, 1984.

[7] O.V. Chernoyarov, Sai Si Thu Min, A.V. Salnikova, B.I. Shakhtarin, A.A. Artemenko, "Application of the Local Markov Approximation Method for the Analysis of Information Processes Processing Algorithms with Unknown Discontinuous Parameters", Applied Mathematical Sciences, vol. 8, No. 90, 2014, pp. 4469-4496.

[8] O.V. Chernoyarov, A.V. Salnikova, A.E. Rozanov, M. Marcokova, "Statistical Characteristics of the Magnitude and Location of the Greatest Maximum of Markov Random Process with Piecewise Constant Drift and Diffusion Coefficients", Applied Mathematical Sciences, vol. 8 , No. 147, 2014, pp. 7341-7357.

[9] E.B. Dynkin, Theory of Markov Processes. Dover Publications Inc., New York, 2006. 